\title{
Novel Accelerating Life Test Method and Its Application by Combining Constant Stress and Progressive Stress
}

Wen-Hua Chen ${ }^{1 *} \mathbb{D}$, Fan Yang ${ }^{1,2}$, Ping Qian ${ }^{1}$, Jun Pan ${ }^{1}$ and Qing-Chuan $\mathrm{He}^{1}$

\begin{abstract}
Constant stress accelerated life tests (ALTS) can be applied to obtain a high estimation accuracy of reliability measurements, but these are time-consuming tests. Progressive stress ALTs can yield failures more quickly but cannot guarantee the estimation accuracy of reliability measurements. In this paper, a progressive-constant combination stress ALT is proposed to combine the merits of both tests. The optimal plan, in which the design variables are the initial progressive stress level, the progressive stress ramp rate, the sample allocation proportion of the progressive stress and the constant stress level, is determined using the principle of minimizing the asymptotic variance of the maximum likelihood estimator of the natural log reliable life for the connectors. A comparison between the optimal PCCSALT plan and the CSALT plan with the same sample size and estimation accuracy shows that the test time is reduced by $13.59 \%$ by applying the PCCSALT.
\end{abstract}

Keywords: Constant stress, Progressive stress, Accelerated life test, Optimal test plan, Reliability test

\section{Introduction}

Accelerated life tests (ALTs) can yield life information of a product in a short time [1-3]. Constant stress accelerated life tests (CSALTs) have the advantages of theory maturity and high statistical precision, but they require many samples and a long test time. Progressive stress accelerated life tests (PSALTs) [4] can generate results in a shorter test time and require a smaller sample size, but they are not widely used in practice because of the immature statistical method and the poor estimation accuracy [5, 6]. To yield accurate estimates of reliability measurements (e.g., reliable life, hazard rate), many studies have been performed on the design of an optimum PSALT plan have. Prot [7] proposed the PSALT optimal design method for a Weibull distribution. Bai et al. [8] optimized a time-censored simple ramp stress test plan for the power-Weibull model and then proposed an optimum single ramp stress test plan [9], which is more

\footnotetext{
*Correspondence: chenwh@zstu.edu.cn

1 Zhejiang Province's Key Laboratory of Reliability Technology for Mechanical and Electronic Product, Zhejiang Sci-Tech University, Hangzhou 310018, China

Full list of author information is available at the end of the article
}

accurate and efficient than the plan in Ref. [8]. Liao and Elsayed [10] developed an optimum method for the lognormal distribution. Ma and Meeker [11] presented a new approach for computing approximate variances and used it to convert and optimize the plan in a previous study [10]. Zhu and Elsayed [12] developed an optimal model for exponential distribution with time censored and progressive stress based on the cumulative exposure model. Srivastava and Mittal [13] developed the model of the optimum multi-objective PSALT with a stress upper bound for a Burr type-XII distribution under time censoring. These studies have demonstrated that the design of the optimum test plan can contribute to improving the life estimation accuracy, but the improvement degree is not obvious.

To synthesize the characteristics of the CSALT and PSALT, a progressive-constant combination stress ALT (PCCSALT) is proposed. The example of the Y11X-1832 electrical connector is used to demonstrate the new test method. In the test plan optimization, the minimum asymptotic variance of the maximum likelihood (ML) estimator of the reliable life at design stress is treated as the optimality principle. The initial stress level, the stress 
ramp rate, the sample allocation proportion of progressive stress and the constant stress level should be determined to improve the test efficiency and maintain the same estimation accuracy as that of the CSALT.

\section{Statistical Model of the Electrical Connectors}

The life of electrical connectors $t$ basically depends on the temperature and follows a two-parameter Weibull distribution under storage and working conditions [14]. The cumulative distribution function (CDF) of $t$ is

$$
F(t)=1-\exp \left[-(t / \eta)^{m}\right]
$$

where $m$ is the shape parameter, $\eta$ is the characteristic life.

Generally, the reliability of the electrical connector is represented with the $P$ th quartile life at a certain working temperature. If failure mechanisms that expose at different temperature stresses are the same as those that expose in field use, then the statistical model of the electrical connector can be expressed as follows [14].

(1) The product lifetime is statistically independent and follows a two-parameter Weibull distribution.

(2) The parameter $m$ is a constant at different temperature stresses.

(3) The relationship between $\eta$ and the working temperature, $T$, satisfies the Arrhenius model, namely,

$$
\eta=A \exp (\Delta E / k T)
$$

where $A$ is the constant, $\Delta E$ is the activation energy for the reaction $(\mathrm{eV}), k$ is Boltzmann's constant, $k=0.8617 \times 10^{-4} \mathrm{eV} / \mathrm{K}, T$ is the thermodynamic temperature $(\mathrm{K})$.

If $y$ equals the logarithmic lifetimes, $\ln t$, then Eq. (1) can be transformed into an extreme value distribution from a Weibull distribution. The CDF is

$$
F(y)=1-\exp \{-\exp [(y-\mu) / \sigma]\},
$$

where $\mu$ is the location parameter, $\mu=\ln \eta, \sigma$ is the scale parameter, $\sigma=1 / m$.

Therefore, the Arrhenius-Weibull model described by Eq. (1) and Eq. (2) can be transformed into the linear extreme value model shown in Eq. (3), which can be described as follows:

(1) $y$ is statistically independent and follows an extreme value distribution.

(2) $\sigma$ is a constant and independent of the stress.

(3) $\mu$ is a function of the stress (generally, the transformed stresses) $x$, that is,

$\ln \eta=\mu(x)=\gamma_{0}+\gamma_{1} x$, where $\gamma_{0}=\ln A, \gamma_{1}=\Delta E / 10^{3} k, x=10^{3} / T$.

To simplify the calculation and make the results more intuitive, the transformed stress should be standardized, as follows:

$$
\xi(x)=\frac{x-x_{0}}{x_{m}-x_{0}},
$$

where $x_{0}$ is the normal working stress level, $x_{\mathrm{m}}$ is the highest test stress level.

The normal working temperature, $T_{0}$, corresponds to $\xi_{0}=0$, whereas the highest test temperature, $T_{\mathrm{m}}$, corresponds to $\xi_{\mathrm{m}}=0$. The $\log$ scale parameter $\ln \eta$ is

$$
\ln \eta=\mu(\xi)=\beta_{0}+\beta_{1} \xi,
$$

where $\beta_{0}=\gamma_{0}+\gamma_{1} x_{0}, \beta_{1}=\left(x_{\mathrm{m}}-x_{0}\right) \gamma_{1}, \xi$ is the standardized stress.

The discussions below are based on Eq. (4).

\section{Design Principle for the PCCSALT Plan \\ 3.1 Stress Loading}

To evaluate the life of the connector under normal stress, the temperature stress is used to conduct the ALT [14]. Figure 1 shows the PCCSALT stress loading profile, where the abscissa denotes $t$, and the ordinate denotes $\xi$.

The plan has two types of temperature stress loadings: a progressive one $\left(\xi_{\mathrm{p}}=\alpha t\right)$ and a constant one $\left(\xi_{\mathrm{c}}\right)$. The temperature stresses range from 0 to 1 . The 0 denotes the normal working stress and the 1 denotes the highest test stress. The parameter $\xi_{\mathrm{p}}$ is the initial value of the progressive stress, and $\alpha$ is the stress ramp rate; $\xi_{\mathrm{c}}$ is a constant stress. The constant stress is always higher than the progressive stress. The parameter $n$ denotes the number of samples, and $\pi_{\mathrm{p}}$ denotes the allocation proportion of the samples at the progressive stress. The test continues until time $\tau$.

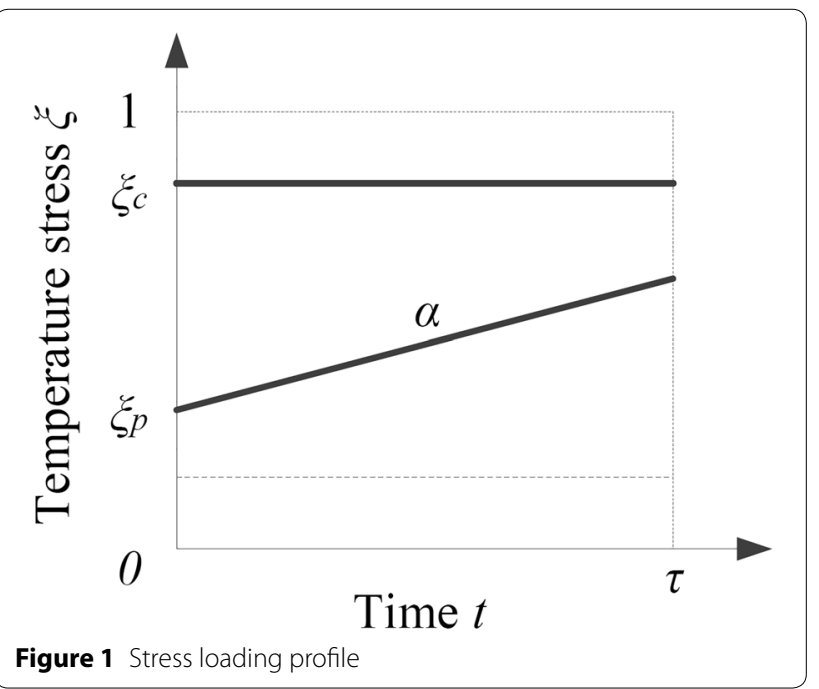




\subsection{Test Design Criterion}

To optimize the test plan, suppose that

(1) The test is stopped at $\tau$ or when all the samples fail;

(2) $T_{m}$ is determined based on the principle of not changing the failure mechanism; and

(3) To guarantee quick failures, the test stress should be higher than the normal working stress.

According to Ref. [15], a higher upper test temperature limit will provide a small asymptotic variance of the ML estimator of the $P$ th quantile of the natural logarithm life. Therefore, $T_{\mathrm{m}}$ should be as high as possible.

\subsection{Test Plan Optimization Criterion}

To improve the life estimation accuracy and balance the test efficiency, the PCCSALT plan could be optimized by using the criteria of minimizing the asymptotic variance of the ML estimator of the Pth quantile of the natural logarithm life.

At stress $\xi_{0}$, the $P$ th quantile of the linear extreme value model is

$$
y_{P}\left(\xi_{0}\right)=\mu\left(\xi_{0}\right)+z_{P} \sigma=\beta_{0}+\beta_{1} \xi_{0}+z_{P} \sigma,
$$

where $z_{P}$ is the $P$ th quantile of the standard extreme value distribution.

Here $P=0.01$ [16], the corresponding quantile of the product is the log reliable life with a reliability of $99 \%$.

\subsection{Estimation Method}

There are many methods for analyzing censored data such as ML methods, linear estimation methods based on order statistics and graphical estimation methods. The ML method is the most widely used because of the following reasons [16]:

(1) Analyzing the censored data by linear estimation methods causes a loss of test information, and these method are not statistically rigorous. However, ML methods are exactly correct.

(2) The optimal test plan obtained by ML methods is close to that obtained using the other estimation methods, and ML methods are easy to apply in plan optimization.

(3) The standard deviation of an ML estimator has the minimum value relative to the others.

Therefore, the ML method is used to compute the Pth quantile and its variance of the product when optimizing ALT plans.

Before optimizing the test plans for the PCCSALT, the model parameters $\beta_{0}, \beta_{1}$ and $\sigma$ must be known. The values of the parameters are usually estimated by experience, similar product data or the results of an early test. In this manner, the optimal test plans may not be optimum, but it is assured that a crude estimator is better than no estimator.

\section{Statistical Analysis Method for Designing the Plan}

\subsection{Sample Likelihood Function from a Constant Stress}

In the CSALT, suppose sample $i$ fails at $t_{i} \leq \tau$. Then, for the linear extreme value model, its likelihood function is

$$
L_{\mathrm{c} i}^{\prime}=\ln f\left(y_{i}\right)=-\ln \sigma+\left(y_{\mathrm{c} i}-\mu\right) / \sigma-\exp \left[\left(y_{\mathrm{c} i}-\mu\right) / \sigma\right],
$$

where $y_{\mathrm{c} i}=\ln t_{i}$.

Suppose sample $i$ survives at $\tau$. It is right censored, and its failure time is greater than $\tau$. The likelihood function is

$$
L_{\mathrm{c} i}^{\prime \prime}=\ln R_{\mathrm{c}}\left(y_{\tau}\right)=-\exp \left[\left(y_{\tau}-\mu\right) / \sigma\right],
$$

where $R_{\mathrm{c}}\left(y_{\tau}\right)$ is the reliability of the sample from constant stress at $\tau, y_{\tau}=\ln \tau$.

Define an indicator function $I_{i}$ such that $I_{i}=1$ if $t_{i} \leq \tau$ and $I_{i}=0$ if $t_{i}>\tau$. Let $z_{\mathrm{c} i}=\left(y_{\mathrm{c} i}-\mu\right) / \sigma$ and $z_{\mathrm{c} \tau}=\left(y_{\tau}-\mu\right) / \sigma$. The log likelihood function for sample $i$ could be expressed as

$$
\begin{aligned}
L_{\mathrm{c} i} & =I_{i} L_{\mathrm{c} i}^{\prime}+\left(1-I_{i}\right) L_{\mathrm{c} i}^{\prime \prime} \\
& =I_{i}\left[-\ln \sigma+z_{\mathrm{c} i}-\exp \left(z_{\mathrm{c} i}\right)\right]+\left(1-I_{i}\right)\left[-\exp \left(z_{\mathrm{c} \tau}\right)\right] .
\end{aligned}
$$

For $n_{\mathrm{c}}$ samples tested under constant stress, the sample $\log$ likelihood function is

$$
L_{\mathrm{c}}=\sum_{i=1}^{n_{\mathrm{c}}} L_{\mathrm{c} i} .
$$

\subsection{PSALT Theory and Statistical Analysis Method \\ 4.2.1 PSALT Theory}

The detailed form of the life distribution for the electrical connectors under progressive stress is derived based on the cumulative exposure model [16]. This model can be expressed as follows [17]: the remaining life of the sample only subject to the current stress and the current cumulative portion failed, regardless of how the portion accumulated. The model ignores the effect of the change in stress. For the samples under varying stress (e.g., the step stress, the progressive stress), at a given time, the failure probability of a survivor is according to the cumulative distribution for the stress at that time, but beginning when the previously accumulated portion failed [18]. The determination of starting time follows the equivalence 
principle in which the same cumulative exposure means the same failure probability.

In the PSALT, the stress $\xi$ has a relationship with $t$.

$$
\xi(t)=\xi_{p}+\alpha t
$$

The progressive stress is considered a limit of the step stress. The progressive stress above can be approximated to a step stress that has $K$ equally spaced test stresses with equal time shifts [19], as shown in Figure 2.

$$
\xi_{i}=\xi_{\mathrm{p}}+(i-0.5) \cdot \alpha \cdot \tau / K, \quad i=1,2, \ldots, K .
$$

Step 1 runs at stress $\xi_{1}$, starting at time 0 , and stops at $\Delta t$. According to Eq. (1), the CDF of the samples could be written as

$$
F(t)=1-\exp \left\{-\left[t / \eta\left(\xi_{1}\right)\right]^{m}\right\}, \quad 0 \leq t<\Delta t .
$$

Step 2 runs at stress $\xi_{2}$, and its equivalent start time is determined by the cumulative exposure at stress $\xi_{1}$. On the basis of the equivalence principle, the equivalent start time $t_{1}$ is just the solution of

$$
1-\exp \left\{-\left[t_{1} / \eta\left(\xi_{2}\right)\right]^{m}\right\}=1-\exp \left\{-\left[\Delta t / \eta\left(\xi_{1}\right)\right]^{m}\right\} .
$$

Therefore, $t_{1}$ equals to $\eta\left(\xi_{2}\right) \Delta t / \eta\left(\xi_{1}\right)$ at stress $\xi_{2}$. Then, the $\mathrm{CDF}$ of the samples at stress $\xi_{2}$ by total time $t$ is

$$
F(t)=1-\exp \left\{-\left[\left(t-\Delta t+t_{1}\right) / \eta\left(\xi_{2}\right)\right]^{m}\right\}, \quad \Delta t \leq t<2 \Delta t .
$$

In general, the equivalent start time for step $i$ is given by $t_{i-1}=\eta\left(\xi_{i}\right) \sum_{j=1}^{i-1} \Delta t / \eta\left(\xi_{j}\right)$ at stress $\xi_{i}$. The CDF of the samples at stress $\xi_{i}$ by total time $t$ is

$$
F(t)=1-\exp \left\{-[\varepsilon(t)]^{m}\right\}, \quad(i-1) \Delta t \leq t<i \Delta t .
$$

where $\varepsilon(t)$ is the cumulative exposure,

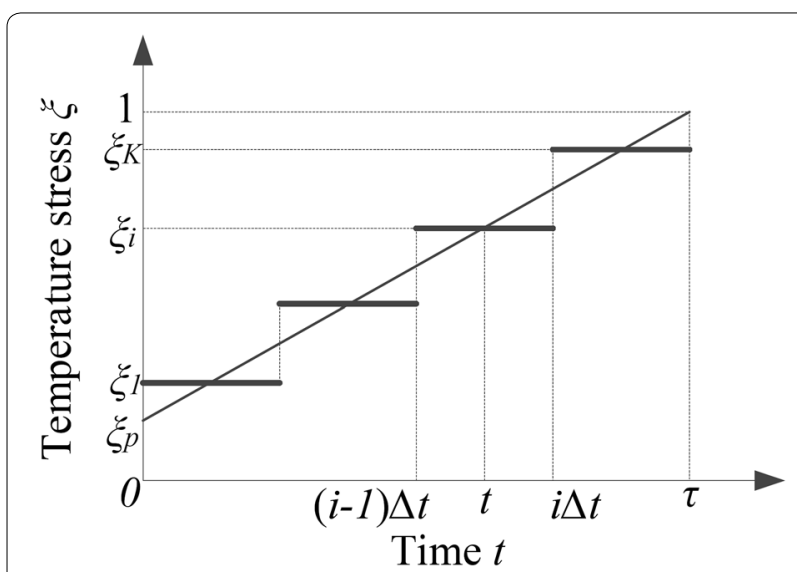

Figure 2 Approximation of a progressive stress by a step stress

$$
\varepsilon(t)=\frac{t-(i-1) \Delta t}{\eta\left(\xi_{i}\right)}+\sum_{j=1}^{i-1} \frac{\Delta t}{\eta\left(\xi_{j}\right)}
$$

As $K \rightarrow \infty, i \rightarrow \infty$. For an infinitesimal time $\mathrm{d} t$, $t-(i-1) \Delta t \rightarrow \mathrm{d} t, \Delta t \rightarrow \mathrm{d} t$. At $t$, the stress can be described as $\xi(t)$. Then,

$$
\begin{aligned}
\lim _{i \rightarrow \infty} \varepsilon(t) & =\lim _{i \rightarrow \infty}\left[\frac{t-(i-1) \Delta t}{\eta\left(\xi_{i}\right)}+\sum_{j=1}^{i-1} \frac{\Delta t}{\eta\left(\xi_{j}\right)}\right] \\
& =\int_{(i-1) \Delta t}^{t} \frac{\mathrm{d} t}{\eta[\xi(t)]}+\int_{0}^{(i-1) \Delta t} \frac{\mathrm{d} t}{\eta[\xi(t)]} \\
& =\int_{0}^{t} \frac{\mathrm{d} t}{\eta[\xi(t)]}
\end{aligned}
$$

Thus, the cumulative exposure as a sum (formed under the step stress) transforms into an integral (formed under the progressive stress). At the progressive stress, the cumulative exposure is

$$
\begin{aligned}
\varepsilon(t) & =\int_{0}^{t} \frac{\mathrm{d} t}{\eta[\xi(u)]}=\int_{0}^{t} \frac{1}{\exp \left[\beta_{0}+\beta_{1} \xi(t)\right]} \mathrm{d} u \\
& =\frac{1-\exp \left(-\beta_{1} \alpha t\right)}{\beta_{1} \alpha \exp \left(\beta_{0}+\beta_{1} \xi_{p}\right)} .
\end{aligned}
$$

If a sample fails at $t$, then Eq. (9) is its cumulative exposure. Based on the equivalence principle, the equivalent time at initial stress $\xi_{\mathrm{p}}$ is

$$
t_{\mathrm{p}}=\varepsilon(t) \cdot \eta\left(\xi_{\mathrm{p}}\right)=\left[1-\exp \left(-\beta_{1} \alpha t\right)\right] / \beta_{1} \alpha,
$$

where $\eta\left(\xi_{\mathrm{p}}\right)$ is the characteristic life at initial stress $\xi_{\mathrm{p}}$,

$$
\eta\left(\xi_{\mathrm{p}}\right)=\exp \left(\beta_{0}+\beta_{1} \xi_{\mathrm{p}}\right) .
$$

The corresponding failure probability is

$$
F\left(t_{\mathrm{p}}\right)=1-\exp \left\{-\left[t_{\mathrm{p}} / \eta\left(\xi_{\mathrm{p}}\right)\right]^{m}\right\} .
$$

The equation above has proven that after being transformed, the life of the products from the progressive stress still follows the Weibull distribution at the constant stress.

Combining Eqs. (7)-(9), the CDF of the products under progressive stress is

$$
\begin{aligned}
F(t) & =1-\exp \left\{-[\varepsilon(t)]^{m}\right\} \\
& =1-\exp \left\{-\left[\frac{1-\exp \left(-\beta_{1} \alpha t\right)}{\beta_{1} \alpha \exp \left(\beta_{0}+\beta_{1} \xi_{\mathrm{p}}\right)}\right]^{m}\right\} .
\end{aligned}
$$

The probability density function (PDF) is the partial derivative of the CDF with respect to $t$, that is, 


$$
\begin{aligned}
f(t)= & \frac{m}{\exp \left(\beta_{0}+\beta_{1} \xi_{\mathrm{p}}+\beta_{1} \alpha t\right)}\left[\frac{1-\exp \left(-\beta_{1} \alpha t\right)}{\beta_{1} \alpha \exp \left(\beta_{0}+\beta_{1} \xi_{\mathrm{p}}\right)}\right]^{m-1} \\
& \times \exp \left\{-\left[\frac{1-\exp \left(-\beta_{1} \alpha t\right)}{\beta_{1} \alpha \exp \left(\beta_{0}+\beta_{1} \xi_{\mathrm{p}}\right)}\right]^{m}\right\} .
\end{aligned}
$$

\subsubsection{Sample Likelihood Function from the Progressive Stress} Under progressive stress, suppose sample $j$ fails at $t_{j} \leq \tau$. Based on Eq. (11), its likelihood function is

$$
\begin{aligned}
L_{\mathrm{p} j}^{\prime} & =\ln f\left(t_{j}\right) \\
& =-\beta_{1}\left(\xi_{\mathrm{p}}+\alpha t_{j}\right)-y_{\mathrm{p} j}-\ln \sigma+z_{\mathrm{p} j}-\exp \left(z_{\mathrm{p} j}\right),
\end{aligned}
$$

where

$$
\begin{aligned}
& y_{\mathrm{p} j}=\ln c\left(t_{j}\right), \\
& z_{\mathrm{p} j}=\left(y_{\mathrm{p} j}-\beta_{0}\right) / \sigma, \\
& c\left(t_{j}\right)=\left[1-\exp \left(-\beta_{1} \alpha t_{j}\right)\right] /\left[\beta_{1} \alpha \exp \left(\beta_{1} \xi_{\mathrm{p}}\right)\right] .
\end{aligned}
$$

Suppose sample $j$ survives at $\tau$. It is right censored, and its failure time is greater than $\tau$. The likelihood function is

$$
L_{\mathrm{p} j}^{\prime \prime}=\ln R_{\mathrm{p}}(\tau)=-\exp \left(z_{\mathrm{p} \tau}\right)
$$

where $R_{\mathrm{p}}(\tau)$ is the reliability of the sample from progressive stress at $\tau, z_{\mathrm{p} \tau}=\left[\ln c(\tau)-\beta_{0}\right] / \sigma$.

Define an indicator function $I_{j}$ such that $I_{j}=1$ if $t_{j} \leq \tau$ and $I_{j}=0$ if $t_{j}>\tau$. The log likelihood function for sample $j$ is

$$
\boldsymbol{F}=\left[\begin{array}{lll}
E\left(-\partial^{2} L / \partial \beta_{0}^{2}\right) & E\left(-\partial^{2} L / \partial \beta_{0} \partial \beta_{1}\right) & E\left(-\partial^{2} L / \partial \beta_{0} \partial \sigma\right) \\
E\left(-\partial^{2} L / \partial \beta_{0} \partial \beta_{1}\right) & E\left(-\partial^{2} L / \partial \beta_{1}^{2}\right) & E\left(-\partial^{2} L / \partial \beta_{1} \partial \sigma\right) \\
E\left(-\partial^{2} L / \partial \beta_{0} \partial \sigma\right) & E\left(-\partial^{2} L / \partial \beta_{1} \partial \sigma\right) & E\left(-\partial^{2} L / \partial \sigma^{2}\right)
\end{array}\right]
$$

According to Eq. (13),

$$
\begin{aligned}
\boldsymbol{F} & =\frac{1}{\sigma^{2}}\left[\sum_{i=1}^{\left(1-\pi_{\mathrm{p}}\right) n}\left(\begin{array}{lll}
A_{1 i} & \xi_{\mathrm{c}} A_{1 i} & A_{2 i} \\
\xi_{\mathrm{c}} A_{1 i} & \xi_{\mathrm{c}}^{2} A_{1 i} & \xi_{c} A_{2 i} \\
A_{2 i} & \xi_{\mathrm{c}} A_{2 i} & A_{3 i}
\end{array}\right)+\sum_{i=1}^{\pi_{\mathrm{p}} n}\left(\begin{array}{lll}
B_{1} & B_{2} & B_{3} \\
B_{2} & B_{4} & B_{5} \\
B_{3} & B_{5} & B_{6}
\end{array}\right)\right] \\
& =\frac{n}{\sigma^{2}}\left(\begin{array}{lll}
\left(1-\pi_{\mathrm{p}}\right) A_{1}+\pi_{\mathrm{p}} B_{1} & \left(1-\pi_{\mathrm{p}}\right) \xi_{\mathrm{c}} A_{1}+\pi_{\mathrm{p}} B_{2} & \left(1-\pi_{\mathrm{p}}\right) A_{2}+\pi_{\mathrm{p}} B_{3} \\
\left(1-\pi_{\mathrm{p}}\right) \xi_{\mathrm{c}} A_{1}+\pi_{\mathrm{p}} B_{2} & \left(1-\pi_{\mathrm{p}}\right) \xi_{\mathrm{c}}^{2} A_{1}+\pi_{\mathrm{p}} B_{4} & \left(1-\pi_{\mathrm{p}}\right) \xi_{\mathrm{c}} A_{2}+\pi_{\mathrm{p}} B_{5} \\
\left(1-\pi_{\mathrm{p}}\right) A_{2}+\pi_{\mathrm{p}} B_{3} & \left(1-\pi_{\mathrm{p}}\right) \xi_{\mathrm{c}} A_{2}+\pi_{\mathrm{p}} B_{5} & \left(1-\pi_{\mathrm{p}}\right) A_{3}+\pi_{\mathrm{p}} B_{6}
\end{array}\right) .
\end{aligned}
$$


where $\quad A_{1}=1-\exp \left[-\exp \left(z_{\mathrm{c} \tau}\right)\right], \quad A_{2}=\int_{-\infty}^{z_{\mathrm{c} \tau}} z \exp (2 z)$ $\exp [-\exp (z)] d z+z_{\mathrm{c} \tau} \exp \left[z_{\mathrm{c} \tau}-\exp \left(z_{\mathrm{c} \tau}\right)\right]$,

$$
\begin{aligned}
A_{3}= & \int_{-\infty}^{z_{\mathrm{c} \tau}} z \exp (z) \exp [-\exp (z)][z \exp (z)+ \\
\exp (z)-1] d z+z_{\mathrm{c} \tau}\left(z_{\mathrm{c} \tau}+1\right) \exp \left[z_{\mathrm{c} \tau}-\exp \left(z_{\mathrm{c} \tau}\right)\right] & \\
B_{1}= & 1-\exp \left[-\exp \left(z_{\mathrm{p} \tau}\right)\right], \\
B_{2}= & -\int_{0}^{\tau} \exp (z) y^{\prime} f(t) d t-y_{\tau}^{\prime} \exp \left[z_{\mathrm{p} \tau}-\exp \left(z_{\mathrm{p} \tau}\right)\right] \\
B_{3}= & \int_{0}^{z_{\mathrm{p} \tau}} z \exp (z) \exp [z-\exp (z)] d z+z_{\mathrm{p} \tau} \exp \left[z_{\mathrm{p} \tau}-\exp \left(z_{\mathrm{p} \tau}\right)\right] \\
B_{4}= & \int_{0}^{\tau}\left\{\sigma y^{\prime \prime}[\exp (z)+\sigma-1]+y^{\prime 2} \exp (z)\right\} f(t) d t+ \\
& \left(y_{\tau}^{\prime 2}+\sigma y^{\prime \prime}{ }_{\tau}\right) \exp \left[z_{\mathrm{p} \tau}-\exp \left(z_{\mathrm{p} \tau}\right)\right]
\end{aligned}
$$$$
B_{5}=\int_{0}^{\tau} y^{\prime}[1-\exp (z)-z \exp (z)] f(t) d t-
$$$$
y_{\tau}^{\prime}\left(1+z_{\mathrm{p} \tau}\right) \exp \left[z_{\mathrm{p} \tau}-\exp \left(z_{\mathrm{p} \tau}\right)\right] \text {, }
$$$$
B_{6}=\int_{0}^{z_{\mathrm{p} \tau}} z^{2} \exp (z) \exp [z-\exp (z)] d z+B 1+
$$$$
z_{\mathrm{p} \tau}^{2} \exp \left[z_{\mathrm{p} \tau}-\exp \left(z_{\mathrm{p} \tau}\right)\right]
$$

$z_{\mathrm{c} \tau}=(\ln \tau-\mu) / \sigma, \mu=\ln \eta\left(\xi_{\mathrm{c}}\right), z=\left(y-\beta_{0}\right) / \sigma, y=c(t)$,

$c(t)=\left[1-\exp \left(-\beta_{1} \alpha t\right)\right] /\left[\beta_{1} \alpha \exp \left(\beta_{1} \xi_{\mathrm{p}}\right)\right]$,

$z_{\mathrm{p} \tau}=\left[\ln c(\tau)-\beta_{0}\right] / \sigma$,

$c(\tau)=\left[1-\exp \left(-\beta_{1} \alpha \tau\right)\right] /\left[\beta_{1} \alpha \exp \left(\beta_{1} \xi_{\mathrm{p}}\right)\right]$,

$f(t)=\frac{m}{\exp \left(\beta_{0}+\beta_{1} \xi_{\mathrm{p}}+\beta_{1} \alpha t\right)}\left[\frac{1-\exp \left(-\beta_{1} \alpha t\right)}{\beta_{1} \alpha \exp \left(\beta_{0}+\beta_{1} \xi_{\mathrm{p}}\right)}\right]^{m-1} \times$

$$
\exp \left\{-\left[\frac{1-\exp \left(-\beta_{1} \alpha t\right)}{\beta_{1} \alpha \exp \left(\beta_{0}+\beta_{1} \xi_{\mathrm{p}}\right)}\right]^{m}\right\} \text {, }
$$

$y^{\prime}=\alpha t /\left[\exp \left(\beta_{1} \alpha t\right)-1\right]-1 / \beta_{1}-\xi_{\mathrm{p}}$,

$y_{\tau}^{\prime}=\alpha \tau /\left[\exp \left(\beta_{1} \alpha \tau\right)-1\right]-1 / \beta_{1}-\xi_{\mathrm{p}}$,

$y^{\prime \prime}=1 / \beta_{1}^{2}-\alpha^{2} t^{2} \exp \left(\alpha \beta_{1} t\right) /\left[\exp \left(\alpha \beta_{1} t\right)-1\right]^{2}$,

$y_{\tau}^{\prime \prime}=1 / \beta_{1}^{2}-\alpha^{2} \tau^{2} \exp \left(\alpha \beta_{1} \tau\right) /\left[\exp \left(\alpha \beta_{1} \tau\right)-1\right]^{2}$.

\subsection{Estimate of the Function Variance}

Based on Eq. (5), for the linear extreme value model, the ML estimate of the Pth quantile $\hat{y}_{P}\left(\xi_{0}\right)$ at the normal working stress is a function of $\hat{\beta}_{0}, \hat{\beta}_{1}$ and $\hat{\sigma}$, that is,

$$
\hat{y}_{P}\left(\xi_{0}\right)=\hat{\beta}_{0}+\hat{\beta}_{1} \xi_{0}+z_{P} \hat{\sigma} .
$$

Since $\hat{y}_{P}\left(\xi_{0}\right)$ is close to a normal distribution [16], the asymptotic variance for $\hat{y}_{P}\left(\xi_{0}\right)$ is

$$
\operatorname{Avar}\left[\hat{y}_{P}\left(\xi_{0}\right)\right]=\left(1,0, z_{P}\right) \boldsymbol{F}^{-1}\left(1,0, z_{P}\right)^{\mathrm{T}}=\sigma^{2} V / n,
$$

\section{Model for Designing the Optimal Plan}

\subsection{Objective Function}

The values of $T_{0}, T_{\mathrm{m}}$, and $\tau$ are given before planning the optimal PCCSALT. The initial estimators of the model parameters $\hat{\beta}_{0}, \hat{\beta}_{1}$ and $\hat{\sigma}$ are determined by experience, similar product data or the results of an early test. The value of $n$ is determined by $V$ and $\hat{\sigma}$. Thus, the ML estimator $V$ is treated as the objective function according to Eq. (16) and the criteria of minimizing $\operatorname{Avar}\left[\hat{y}_{P}\left(\xi_{0}\right)\right]$.

\subsection{Design Variables}

Since $T_{0}, T_{\mathrm{m}}, \hat{\beta}_{0}, \hat{\beta}_{1}$ and $\hat{\sigma}$ are given, $V$ depends on $\xi_{\mathrm{p}}, \alpha$, $\pi_{\mathrm{p}}$, and $\xi_{\mathrm{c}}$. Therefore, the design variables are

$\xi_{\mathrm{p}}, \alpha, \pi_{\mathrm{p}}, \xi_{\mathrm{c}}$.

\subsection{Constraints}

(1) The value of $\xi_{\mathrm{c}}$ should range from 0 to 1 , namely, $0 \leq \xi_{\mathrm{c}} \leq 1$

(2) Considering the test design principle the progressive stress should always be less than the constant stress to ensure quick failures for the constant stress. That is,

$\xi_{\mathrm{p}}+\alpha t \leq \xi_{\mathrm{c}}$.

(3) $\xi_{\mathrm{p}}$ and $\alpha$ are chosen to guarantee that the progressive stress values range from 0 to 1 . Moreover, to ensure enough failures for the progressive stress, the progressive initial stress should be set $20 \%$ higher than the normal working stress, that is,

$0.2 \leq \xi_{\mathrm{p}}+\alpha t \leq 1$.

(4) The value of $\pi_{\mathrm{p}}$ should also be set to range from 0 to 1. To make the plan feasible, two stresses should be allocated to a certain amount of samples. Set the $\pi_{\mathrm{p}}$ values to range between 0.3 and 0.7 ,

$0.3 \leq \pi_{\mathrm{p}} \leq 0.7$

\subsection{Sample Size}

Based on the ML theory, the MLE of the Pth quantile of $\hat{y}_{P}\left(\xi_{0}\right)$ under normal working stress is close to the normal distribution, with a mean of $\hat{y}_{P}\left(\xi_{0}\right)$ and a variance of $\operatorname{Var}\left[y_{P}\left(\xi_{0}\right)\right]$. If $\hat{y}_{P}\left(\xi_{0}\right)$ replaces $y_{P}\left(\xi_{0}\right)$, then the confidence bounds of $\hat{y}_{P}\left(\xi_{0}\right)$ for confident values $\gamma$ are

$$
\left[y_{P}\left(\xi_{0}\right)\right]_{\mathrm{U}}=\hat{y}_{P}\left(\xi_{0}\right)+K_{\gamma} \sqrt{\sigma^{2} V / n},
$$

where $V$ is the variance factor for the PCCSALT plan.

$$
\left[y_{P}\left(\xi_{0}\right)\right]_{\mathrm{L}}=\hat{y}_{P}\left(\xi_{0}\right)-K_{\gamma} \sqrt{\sigma^{2} V / n}
$$


where $K_{\gamma}$ is the $(100(1+\gamma) / 2)$ th standard normal quantile.

If $\hat{y}_{P}\left(\xi_{0}\right)$ is within $y_{P}\left(\xi_{0}\right) \pm W$ with probability $\gamma$, then

$$
W=K_{\gamma} \sqrt{\sigma^{2} V / n}
$$

Thus, the sample size $n$ is

$$
n=V\left(K_{\gamma} \sigma / W\right)^{2}
$$

\section{Optimal Design of the Electrical Connectors PCCSALT Plan}

\subsection{Test Parameters}

\subsubsection{Temperature Stress}

The working temperature of the Y11X series electrical connectors is $218.15-401.15 \mathrm{~K}$ according to a previous study [18]. In accordance with the usage conditions of the connectors, $T_{0}=298.15 \mathrm{~K}$ is selected as the normal working temperature.

The electrical contact failure caused by the accumulation of the oxide film is the main mode [14] for the Y11X series electrical connectors. Since the highest test temperature should be close to the upper working temperature limit $(431.15 \mathrm{~K})$ with the requirement of avoiding introducing failure modes that will not be encountered in normal use, $T_{\mathrm{m}}=431.15 \mathrm{~K}$ is chosen as the highest temperature stress in the test.

\subsubsection{Censored Time}

As the connectors fail fast at the progressive stress, considering the censored time for the CSALT [20] and the step stress ALT (SSALT) [21], this paper takes $\tau=1000 \mathrm{~h}$ as the censored time.

\subsubsection{Parameter Estimators for the Statistical Model}

By using the statistical analysis results [14] of the data from the previous CSALT, the crude estimate values of the model parameters for the Y11X-1832 electrical connector are $\hat{\gamma}_{0}=-21.2813, \hat{\gamma}_{1}=9.7579$, and $\hat{\sigma}=0.9867$. Using the transforms in Section 2, the standardized parameters are
Table 2 Optimal CSALT plan

\begin{tabular}{llllll}
\hline No. & $\begin{array}{l}\text { Stress } \boldsymbol{\xi}, \\
\text { Temperature } \\
\boldsymbol{T}(\mathrm{K})\end{array}$ & $\begin{array}{l}\text { Sample } \\
\text { allocation } \\
\text { proportion } \\
\boldsymbol{\pi}\end{array}$ & $\begin{array}{l}\text { Censored } \\
\text { time } \\
\boldsymbol{t}(\mathbf{h})\end{array}$ & $\begin{array}{l}\text { Sample } \\
\text { size } \boldsymbol{n}\end{array}$ & $\begin{array}{l}\text { Variance } \\
\text { factor } \boldsymbol{V}\end{array}$ \\
\hline 1 & $0.4313,338.45$ & 0.7 & 1000 & 40 & 24.1501 \\
2 & $1,436.15$ & 0.3 & & & \\
\hline
\end{tabular}

$$
\hat{\beta}_{0}=11.4467, \hat{\beta}_{1}=-8.0340, \hat{\sigma}=0.9867 \text {. }
$$

6.2 Optimal Results

MATLAB's fmincon solver is used to address the optimization problem. The objective function converges to $V_{\mathrm{m}}=23.6837$, and the corresponding test plan is given in Table 1.

Given the confidence $\gamma=40 \%, K_{\gamma}=0.6745$ and confidence interval width $2 W=0.8$, substitute them into Eq. (17), and the sample size is $n=40$.

A comparison between the PCCSALT plan (see Table 1) and the CSALT plan (see Table 2) is applied to verify that the proposed plan is better, as it has a higher estimation accuracy for $y_{\mathrm{p}}$. The compared simple CSALT plan is defined in the following manner: the mid value of the progressive stress, namely, $\xi_{1}=\xi_{\mathrm{p}}+\alpha \cdot t_{\mathrm{m}} / 2$, is used as the low stress, while the sample allocation proportion, the high stress and the censored time are the same as those in the proposed plan.

In general, the estimation accuracy depends on the sample size and test time. To improve the estimation accuracy, more test time is required if the sample size is fixed. To compare the efficiency of the two plans quantitatively, the test time for the CSALT is elongated to when its variance factor equals that of the proposed plan. Here, $1157 \mathrm{~h}$ are obtained. Therefore, the test time could be shortened by $13.59 \%$ if using the PCCSALT plan under the same requirements of estimation accuracy and sample size.

Table 1 Optimal PCCSALT plan

\begin{tabular}{lllll}
\hline Stress type & $\begin{array}{l}\text { Stress } \boldsymbol{\xi} \text {, Temperature } \\
\boldsymbol{T}(\mathbf{K})\end{array}$ & $\begin{array}{l}\text { Stress change rate } \\
\boldsymbol{a}\left(\mathbf{h}^{-\mathbf{1}}\right)\end{array}$ & $\begin{array}{l}\text { Sample allocation } \\
\text { proportion } \boldsymbol{\pi}\end{array}$ & $\begin{array}{l}\text { Censored time } \\
\boldsymbol{t}(\mathbf{h})\end{array}$ \\
\hline Progressive & $0.3048,322.26$ & $2.5298 \times 10^{-4}$ & 0.7000 & 1000 \\
Constant & $1,436.15$ & - & 0.3000 & 23.6837 \\
\hline
\end{tabular}




\section{Conclusions}

(1) An PCCSALT method combining progressive stress and constant stress is proposed.

(2) With the same estimation accuracy and sample size, the time of the optimal PCCSALT plan is $13.59 \%$ less than that of the optimal CSALT plan.

\section{Authors' Contributions}

$\mathrm{W}-\mathrm{HC}$ was in charge of the whole trial; FY wrote the manuscript; $\mathrm{PQ}, \mathrm{JP}$ and Q-CH assisted with sampling and laboratory analyses. All authors read and approved the final manuscript.

\section{Author Details}

1 Zhejiang Province's Key Laboratory of Reliability Technology for Mechanical and Electronic Product, Zhejiang Sci-Tech University, Hangzhou 310018, China.

${ }^{2}$ School of Engineering, Huzhou University, Huzhou 313000, China.

\section{Authors' Information}

Wen-Hua Chen, born in 1963, is currently a professor at Zhejiang University and Zhejiang Sci-Tech University, China. He is also the Director of Zhejiang Province's Key Laboratory for Reliability Technology of Mechanical and Electrical Products at Zhejiang Sci-Tech University, China. He received his PhD degree in mechanical manufacturing from Zhejiang University, China, in 1997. He is mainly engaged in research on reliability design, testing, and statistical analysis.

Fan Yang, born in 1987, is a Master-Doctor combined program graduate student of Faculty of Mechanical and Automatic Control, Zhejiang Sci-Tech University, China. Her main interest areas are accelerated life tests and product life predictions and reliability assessments.

Ping Qian, born in 1983, is currently an associate professor at Faculty of Mechanical and Automatic Control, Zhejiang Sci-Tech University, China. She received her PhD degree from Zhejiang University, China, in 2010. Her research interests include the modeling and statistical analysis of accelerated life testing, the design of testing plans, and reliability estimation.

Jun Pan, born in 1974, currently a professor at Faculty of Mechanical and Automatic Control, Zhejiang Sci-Tech University, China. He received his PhD degree from Zhejiang Sci-Tech University, China, in 2011. His research interests include the modeling and statistical analysis of accelerated degradation testing, the design of testing plans, and reliability estimation.

Qing-Chuan He, born in 1984, is currently a lecturer at Faculty of Mechanical and Automatic Control, Zhejiang Sci-Tech University, China. He received his PhD degree from Zhejiang Sci-Tech University, China, in 2013. He is mainly engaged in research on reliability design and testing and systems reliability.

\section{Competing Interests}

The authors declare no competing financial interests.

\section{Funding}

Supported by National Natural Science Foundation of China (Grant No.

51405447), and International Science \& Technology Cooperation Program of China (Grant No. 2015DFA71400).

\section{Publisher's Note}

Springer Nature remains neutral with regard to jurisdictional claims in published maps and institutional affiliations.
Received: 11 October 2016 Accepted: 12 September 2018

Published online: 03 October 2018

\section{References}

[1] G B Yang. Accelerated life tests at higher usage rates. IEEE Transactions on Reliability, 2005, 54(1): 53-57.

[2] C H Li, N Fard. Optimum bivariate step-stress accelerated life test for censored data. IEEE Transactions on Reliability, 2007, 56(1): 77-84.

[3] A Q A Masri, A H Ebrahem. Optimum times for step-stress cumulative exposure model using log-logistic distribution with known scale parameter. Austrian Journal of Statistics, 2009, 38(1): 59-66.

[4] P W Srivastava. Optimum accelerated life testing models with time-varying stresses. New Jersey: World Scientific, 2017.

[5] E M Prot. Fatigue testing under progressive loading: a new technique for testing material. Translation WADCTR-52-148, 1952, 9.

[6] K A Doksum, A Hoyland. Models for variable-stress accelerated life testing experiments based on wiener processes and the inverse Gaussian distribution. Technometrics, 1992, 34(1): 74-82.

[7] L Nilsson. Estimation from accelerated life tests with increasing stress. Sweden: Univ. of Umea, 1985

[8] D S Bai, M S Kim, S W Chung. Optimum simple ramp-tests for the Weibull distribution and type-I censoring. IEEE Transactions on Reliability, 1992, 41(3): 407-413.

[9] D S Bai, Y R Chun, M S Cha. Time-censored ramp tests with stress bound for Weibull life distribution. IEEE Transactions on Reliability, 1997, 46(1): 99-107.

[10] HT Liao, E A Elsayed. Equivalent accelerated life testing plans for loglocation-scale distributions. Naval Research Logistics, 2010, 57(5): 472-488.

[11] Y L Hong, H M Ma, W Q Meeker. A tool for evaluating time -varying-stress accelerated life test plans with log-location-scale distributions. IEEE Transactions on Reliability, 2010, 59(4): 620-627.

[12] Y D Zhu, E A Elsayed. Design of equivalent accelerated life testing plans under different stress applications. Quality Technology \& Quantitative Management, 2011, 8(4): 463-478.

[13] P W Srivastava, N Mittal. Optimum multi-objective ramp-stress accelerated life test with stress upper bound for Burr type-XII distribution. IEEE Transactions on Reliability, 2012, 61(4): 1030-1038.

[14] W H Chen. Research on electrical connector reliability test and analysis. Hangzhou: Zhejiang University, 1997. (in Chinese)

[15] W Q Meeker. A comparison of accelerated life test plans for Weibull and lognormal distributions and type I censoring. Technometrics, 1984, 26(2): 157-171.

[16] W Nelson. Accelerated testing: statistical models, test plans, and data analysis. New Jersey: John Wiley \& Sons, 1990.

[17] K Yoshio. Properties of the Weibull cumulative exposure model. Journal of Applied Statistics, 2006, 33(1): 17-34

[18] Commission on Science, Technology, and Industry for National Defense of the PRC. GJB101A-97 connectors, electrical, circular, miniature, quick coupling, environment resistant, general specification for. Beijing: COSTIND, 1997. (in Chinese)

[19] CXu. Research of the loading ways and statistical analysis method for progressive stress accelerated life test. Hangzhou: Zhejiang Sci-Tech University, 2014. (in Chinese)

[20] W H Chen, Y D Cheng. Optimum design of accelerated life test plans for Weibull life distributions. Journal of Mechanical Engineering, 1999, 33(4): 337-342. (in Chinese)

[21] W H Chen, J J Liu, J Pan, et al. Theory \& method for optimum design of accelerated life test plan under step-stress. Journal of Mechanical Engineering, 2010, 46(10): 182-187. (in Chinese) 